\title{
Theoretical Investigation of Reaction Kinetics in the Reduction of Single Hematite Pellet under Pulsating Flow of Hydrogen*
}

\section{By Munekazu OHMI** and Tateo USUI**}

\section{Synopsis}

The fact that the rate of reduction of a hematite pellet under pulsating flow is higher than that under steady flow can be ascribed to the decrease of the mass-transfer resistance in the gas film. The rate of reduction under pulsating flow is calculated by the use an unreacted-core shrinking model for one interface and Kitaura's expression for the mass-transfer coefficient, and the following conclusions are obtained:

(1) The rate of reduction under pulsating flow increases as the frequency and the amplitude of pulsation increase.

(2) Pulsating flow accelerates the rate of reduction particularly in the early stage of reduction and in a high temperature range. This trend coincides qualitatively with the results previously reported.

(3) The optimum values for the pellet diameter and the time-averaged flow rate were estimated.

(4) When the pellet diameter and the flow rate are not significantly large, the effect of pulsating flow increases gradually as the percentage of $\mathrm{N}_{2}$ gas in the binary bulk gas of $\mathrm{H}_{2}-\mathrm{N}_{2}$ increases.

\section{Introduction}

The rate of reduction of iron oxide under pulsating flow is expected to be higher than that under steady flow, because of the acceleration of the mass-transfer process. ${ }^{1-15)}$ The first trial was experimentally made by Schenck and Cloth ${ }^{16)}$ and the effect of pulsating flow was appreciated. Kodama, et al. ${ }^{17)}$ have inferred the effect to be due to the acceleration of masstransfer in the particle as well as that in the gas film from the fact that the effect is slightly different depending on the kinds of iron ore. Yamada and Tsuda ${ }^{18)}$ and Yavoiskií, et al. ${ }^{19)}$ have shown that the rate of reduction under pulsating flow increases as the flow rate decreases or the temperature increases. The acceleration of the reduction rate under pulsating flow may, therefore, be considered to be based upon the acceleration of the mass-transfer rate mainly in the gas film and partly in the particle. ${ }^{20)}$

In the present paper, the rate of reduction of a single hematite pellet under the pulsating flow of $\mathrm{H}_{2}-\mathrm{N}_{2}$ mixture has been calculated by the use of an unreacted-core shrinking model for one interface considering the resistance due to the rate of gas $f l o w^{21-23)}$ and the expression of Kitaura, et al..$^{8)}$ for the mass-transfer coefficient, under the assumption that the acceleration is ascribed only to the increase of the mass-transfer rate in the gas film.

\section{Overall Reduction Rate}

An equation for the rate of reduction under pulsating flow has been derived on the basis of an unreactedcore shrinking model for one interface, considering such three processes as the mass-transfer in the gas film, the intraparticle diffusion, and the interfacial chemical reaction as well as the resistance due to the rate of gas flow. ${ }^{21-23)}$ Before the derivation, the following assumptions were made:

(1) The acceleration of the overall reduction rate under pulsating flow is ascribed only to the increase of the mass-transfer rate in the gas film.

(2) The physical-property values and the other constants under pulsating flow are the same as those under steady flow.

(3) The pellet is hanged so as to be on the centreline of the pipe wherein the flow velocity is fully developed, and the pulsation is transferred perfectly to the pellet.

(4) The system is isothermal, so that there are no natural convection, no heat of chemical reaction, and no increase in heat-transfer under pulsating flow.

(5) The reduction proceeds topochemically in a macroscopic sense, and the pellet diameter does not change during the course of reduction.

In this case the molar rate of hydrogen through the gas film, the rate of gaseous diffusion through the shell of the reduced iron, and the chemical reaction rate at the interface are respectively given ${ }^{21-23)}$ by

$$
\begin{aligned}
& n_{f j}=-4 \pi r_{o}^{2} k_{f j}\left(C_{a j}-C_{o j}\right)(j=s t, p l) \ldots \ldots \ldots \ldots \ldots \ldots \ldots \ldots \ldots \\
& n_{d j}=-4 \pi D_{e}\left(C_{o j}-C_{i j}\right) /\left(1 / r_{i}-1 / r_{o}\right) \quad(j=s t, p l) . .
\end{aligned}
$$
and

$$
n_{c j}=-4 \pi r_{i}^{2} k_{c}(1+1 / K)\left(C_{i j}-C_{e}\right) \quad(j=s t, p l)
$$

where, the subscripts $j=s t, p l$ denote the quantities for steady and pulsating flow. The reduction is considered to proceed as

$$
n_{f j}=n_{d j}=n_{c j} \equiv n_{j} \quad(j=s t, p l)
$$

and the overall reduction rate is also controlled by the resistance due to the rate of gas flow ${ }^{21-23)}$ as follows.

$$
n_{j}=-V_{T}\left(C_{b}-C_{a j}\right) \quad(j=s t, p l)
$$

This approximate analysis based on the average gas concentration $C_{a j}$ is considered to be more satisfactory for pulsating flow than that for steady flow, because of the mixing effect of pulsation. ${ }^{16)}$ After considerable rearrangements, combination of Eqs. (1) through (5) gives

$$
n_{j}=-\frac{1}{k_{f j}^{\prime}}+\frac{r_{o}}{D_{e}\left\{\begin{array}{c}
1 \\
(1-F)^{2}\left(C_{b}-C_{e}\right)
\end{array}\right\}+1} \begin{gathered}
1 \\
k_{c}(1+1 / K)(1-F)^{2 / 3} \\
\quad(j=s t, p l) \ldots \ldots(6)
\end{gathered}
$$

* Originally published in Tetsu-to-Hagané, 59 (1973), 1902, in Japanese. English version received May 7, 1975.

** Department of Metallurgical Engineering, Osaka University, Suita 565. 
where, $F$ is the fractional reduction and $k_{f j}^{\prime}$ is the modified mass-transfer coefficient including the resistance due to the rate of gas flow $4 \pi r_{o}^{2} / V_{T}$ which is given by the followings:

$$
k_{f j}^{\prime}=\left(1 / k_{f j}+4 \pi r_{o}^{2} / V_{T}\right)^{-1} \quad(j=s t, p l)
$$

Combining Eq. (6) and the relation

$$
n_{j}=4 \pi \rho_{p} \frac{d r_{i}^{3}}{d t_{j}}=-4 \pi \rho_{p} r_{o}^{3} \frac{d F}{d t_{j}} \quad(j=s t, p l)
$$

the integration gives the following expression for the reducing time.

$$
\begin{array}{r}
t_{j}=\frac{3 \rho_{p} r_{o}}{C_{b}-C_{e}}\left\{\frac{F}{3 k_{f j}^{\prime}}+\frac{3-3(1-F)^{2 / 3}-2 F}{6 D_{e} / r_{o}}+\frac{1-(1-F)^{1 / 3}}{k_{c}(1+1 / K)}\right\} \\
(j=s t, p l) \ldots \ldots(9)
\end{array}
$$

\section{Mass-transfer Coefficient in the Gas Film under Pulsating Flow}

Additional disturbances such as ultrasonic wave, turbulence, pulsation, and oscillation of the submerged body in a flow field accelerate the heat- and mass-transfer. The theoretical and experimental analyses have been made ${ }^{1-15)}$ on the mass-transfer for a cylinder and for a sphere and semi-empirical correlations are given. This acceleration is considered to be due to the steady secondary flow ${ }^{2,5)}$ in the case of small amplitude oscillating flow $\left(a / r_{o} \ll 1\right)$ around a cylinder and to the reverse flow ${ }^{9}$ in the case of large amplitude pulsating flow $\left(a \mid r_{o}>1\right)$ around a sphere.

Figure 1 shows the important results of the masstransfer correlation for a sphere. Correlation of Mori, et al. ${ }^{9)}$

$$
S h_{p l}=2+0.944\left(a f d_{p} / \nu\right)^{1 / 2}
$$

is restricted to the case of large amplitude and zero

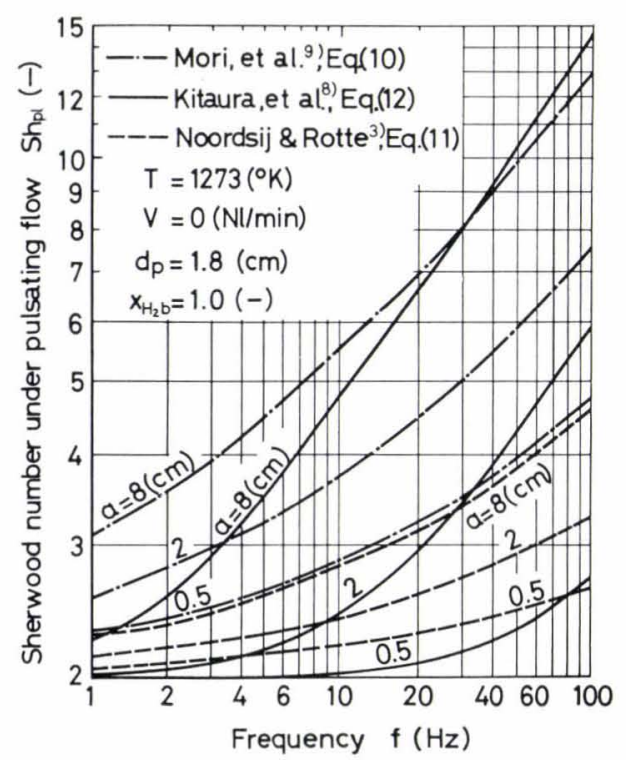

Fig. 1. Mass-transfer from a single sphere under pulsating flow; comparison of Kitaura's expression with others flow rate. Correlation of Noordsij and Rotte ${ }^{3)}$

$$
S h_{p l}=2+0.24 S c^{1 / 2}\left(a f d_{p} / \nu\right)^{1 / 2}
$$

was obtained by the dimensional analysis and by the electrochemical measurement, and is restricted to the case of small amplitude and zero flow rate. Correlation of Kitaura, et al. ${ }^{8)}$

$$
\begin{aligned}
\frac{S h_{p l}}{R e_{p}^{1 / 2}}= & {\left[\left(\frac{2}{R e_{p}^{1 / 2}}+0.55 S c^{1 / 3}\right)^{3}\right.} \\
& \left.+\left(0.35 S c^{1 / 3}\right)^{3}\left(\frac{2 \pi a f}{u}\right)^{2}\left(\begin{array}{c}
3 u \\
2 \pi d_{p} f
\end{array}\right)^{1 / 2}\right]^{1 / 3}
\end{aligned}
$$

was obtained by the hydrodynamic consideration and by the mass-transfer experiments when the sphere was oscillated under steady pipe flow within the ranges of $1 \leqq f \leqq 11 \mathrm{~Hz}, 1 \leqq a \leqq 4 \mathrm{~cm}$, and $40<R e<300$. This correlation is close to Eq. (10) in the case of large amplitude and to Eq. (11) in the case of small amplitude, as shown in Fig. 1. It is not restricted to the case of zero flow rate and is identical with the correlation of Frössling ${ }^{24)}$ for steady flow $(f a=0)$. Hence, the correlation of Kitaura, et al. has been used to estimate the mass-transfer coefficient under pulsating flow, because of the existence of the approximate similarity between oscillation and pulsation. ${ }^{11,25-30)}$ Equation (12) becomes

$$
S h_{p l}=\frac{k_{f p l} d_{p}}{D}=\left[S h_{s t}^{3}+1.17 a^{2} f^{3 / 2} \frac{d_{p}}{D \sqrt{\nu}}\right]^{1 / 3} \ldots
$$

so that $S h_{p l}$ increases in comparison with $S h_{\mathrm{st}}$ as the product $f^{3 / 2} a^{2}$ increses.

The mass-transfer coefficients $k_{f s t}$ and $k_{f p l}$ increase with increasing temperature, and the ratio $k_{f p l} / k_{f s t}$ decreases a little, as shown in Fig. 2 (a). As the timeaveraged flow rate increases, then $k_{f j}$ increases but a little in the case of large pulsation, so that $k_{f p l} / k_{f s t}$ decreases suddenly, as shown in Fig. 2 (b).

Figure 3 shows the variations of the modified masstransfer coefficient $k_{f j}^{\prime}$ and the ratio $k_{f p l}^{\prime} / k_{f s t}^{\prime}$ with

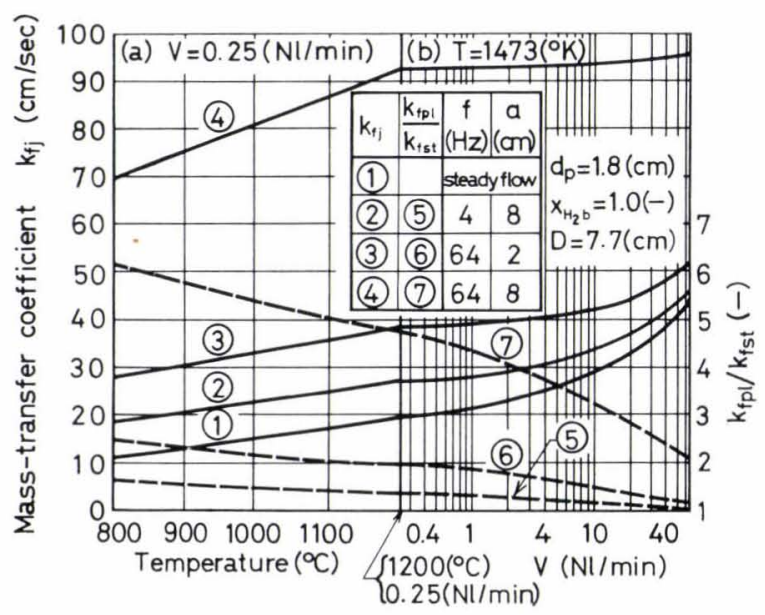

Fig. 2. Mass-transfer coefficients, $k_{f s t}$ and $k_{f p l}$, under steady and pulsating flow respectively, and the ratio $k_{f p l}$ $k_{f s t}$ as a function of (a) temperature $T$ and (b) timeaveraged flow rate $V$

\footnotetext{
* The coefficient 0.478 reported in Eq. (48) of Ref. 9) is in error. The coefficient should be 0.472 .
} 


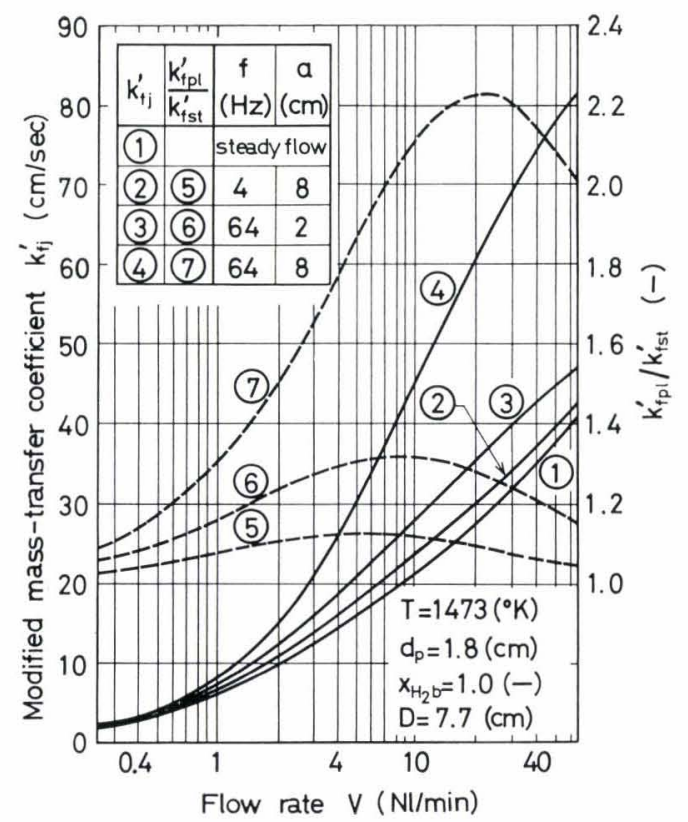

Fig. 3. Modified mass-transfer coefficient

$$
k_{f j}^{\prime}=\left(1 / k_{f j}+4 \pi r_{o}^{2} / V_{T}\right)^{-1} \quad(j=s t, p l)
$$

and the ratio $k_{f p l}^{\prime} / k_{f s t}^{\prime}$ as a function of time-averaged flow rate $V$

flow rate under the same condition as in the case of Fig. 2 (b). As the flow rate increases $k_{f j}^{\prime}$ also increases, but $k_{f p l}^{\prime} / k_{f s t}^{\prime}$ has a maximum value, because of the effect of the resistance due to the rate of gas flow $4 \pi r_{o}^{2} / V_{T}$ in Eq. (7). The optimum flow rate for the acceleration effect of pulsating flow on the reduction may, therefore, be expected.

In the reaction which is influenced only by the mass-transfer process in the gas film, the ratio $k_{f p l} /$ $k_{f s t}$ itself expresses the degree of the acceleration effect of pulsating flow, so that a large effect can be expected. The reduction of a hematite pellet is, however, influenced by the three processes depending on the fractional reduction $F$, so that the effect of pulsating flow is considered not to be so large and to depend on $F$.

\section{Influences of Each Parameter on the Ac- celeration Effect of Pulsating Flow on Re- duction}

Table 1 shows the values of the parameters adopted in the calculation of the reduction process. The reducing conditions were taken from the experimental conditions carried out by Takahashi, et al. ${ }^{31)}$ The degree of approximation of the unreacted-core shrinking model for one interface becomes worse when the temperature is lower than $800^{\circ} \mathrm{C},{ }^{22)}$ or when the porosity is high and moreover the reducing gas $\mathrm{H}_{2}$ is diluted by $\mathrm{N}_{2} \cdot{ }^{32}$ ) Hence, the temperature was taken higher than $800^{\circ} \mathrm{C}$, the influence of porosity $\left(0.05 \leqq \varepsilon_{p} \leqq 0.25\right)$ was examined only for the case of $x_{\mathrm{H}_{2} b}=1.0$, and the general discussions including the influence of mole fraction $\left(0.2 \leqq x_{\mathrm{H}_{2} b} \leqq 1.0\right)$ were made at $\varepsilon_{p}=0.15$.

The reduction curves under steady and pulsating flow are shown in Fig. 4. The reducing time decreases as the frequency and the amplitude of pulsa-
Table 1. Values of the parameters adopted for the calculations of reduction process under steady and pulsating flow conditions

\begin{tabular}{c|l|l|l|l|l|r}
\hline \begin{tabular}{c|c|c|c|c|}
$f$ \\
$(\mathrm{~Hz})$
\end{tabular} & $\begin{array}{c}a \\
(\mathrm{~cm})\end{array}$ & $\begin{array}{c}V \\
(\mathrm{Nl} / \mathrm{min})\end{array}$ & $\begin{array}{c}d_{p} \\
(\mathrm{~cm})\end{array}$ & $\begin{array}{c}\varepsilon_{p} \\
(-)\end{array}$ & $\begin{array}{c}T \\
\left({ }^{\circ} \mathrm{K}\right)\end{array}$ & $\begin{array}{c}x_{\mathrm{H}_{2} b} \\
\left(-{ }^{-}\right)\end{array}$ \\
\hline 0.25 & 0.5 & 0.25 & 0.6 & 0.05 & 1073 & 1.0 \\
1 & 1 & 1 & 1.2 & 0.10 & 1173 & 0.8 \\
4 & 2 & 4 & 1.8 & 0.15 & 1273 & 0.6 \\
16 & 4 & 16 & 2.4 & 0.20 & 1373 & 0.4 \\
64 & 8 & 64 & 3.0 & 0.25 & 1473 & 0.2 \\
\hline
\end{tabular}

Conditions : $\mathrm{D}=7.7(\mathrm{~cm})$, Reducing gas : $\mathrm{H}_{2}-\mathrm{N}_{2}\left(x_{\mathrm{H}_{2} \mathrm{Ob}}=0(-)\right)$, $\rho_{p}=0.0244\left(\mathrm{molFe}_{2} \mathrm{O}_{8} / \mathrm{cm}^{3}\right)$ when $\varepsilon_{p}=0.15(-)$

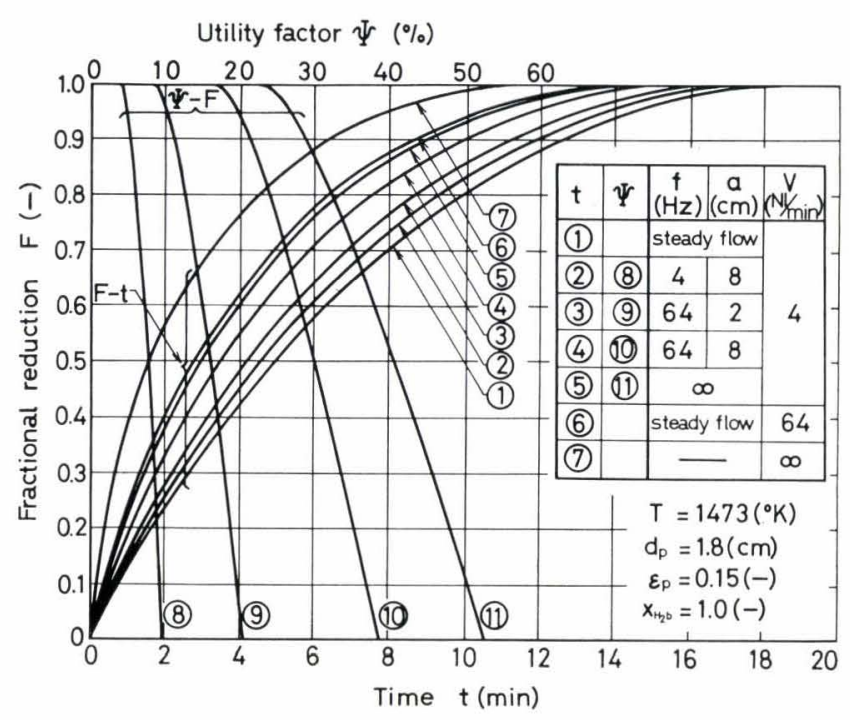

Fig. 4. Reduction curves ( $F-t$ curves) and "utility factor of reduction under pulsating flow" $\Psi$ as a function of fractional reduction $F$ ( $\Psi-F$ curves)

tion increase, and this trend is remarkable especially in the early stage of reduction. The effect of the application of pulsation is similar to that of the increase in the flow rate, as shown by Yavorskii, et al. ${ }^{19)}$

Figure 4 also shows the utility factor of reduction under pulsating flow defined by

$$
\begin{aligned}
& \Psi\left(F ; f, a, V, d_{p}, \varepsilon_{p}, \mathcal{T}, x_{\mathrm{H}_{2} b}\right) \\
& =\frac{t_{s t}\left(F ; V, d_{p}, \varepsilon_{p}, \mathcal{T}, x_{\mathrm{H}_{2} b}\right)-t_{p l}\left(F ; f, a, V, d_{p}, \varepsilon_{p}, T, x_{\mathrm{H}_{2} b}\right)}{t_{s t}\left(F ; V, d_{p}, \varepsilon_{p}, T, x_{\mathrm{H}_{2} b}\right)}
\end{aligned}
$$

as a function of fractional reduction $F$. The abovementioned trends can clearly be understood, so that the influences of each parameter are examined by the use of $\Psi$.

\section{Influence of Flow Conditions}

The influence of the flow conditions on the acceleration effect of pulsating flow on the reduction is considered to be the largest among the several parameters listed in Table 1. A flow field of no turbulence, no free convection, no vibration phenomena, etc. has been assumed in the current work. 


\section{Frequency and Amplitude of Pulsation}

Figure 5 shows the contour lines of $\Psi$ in the $f-a$ domain. From Eq. (13) the values of $k_{f p l}$ are the same when $f^{3 / 2} a^{2}=$ const., i.e.,

$$
3 \log f+4 \log a=\text { const. }
$$

so that the values of $\Psi$ are also the same, the equi- $\Psi$ lines are straight and their inclinations yield $-3 / 4$ in this figure. The utility factor increases as the frequency and the amplitude increase, but the increment becomes small when the product $f^{3 / 2} a^{2}$ becomes large.

\section{Time-averaged Gas Flow Rate}

Figure 6 shows the variation of $\Psi$ with gas flow rate $V$ at given values of the other parameters; the curve (1) is calculated at the standard conditions and the other curves (2) to (7) are calculated at the same conditions as those in the case of (1) except one parameter. In each case, the optimum flow rate seems to exist within the range of 1 to $10 \mathrm{Nl} / \mathrm{min}$. Such a trend is expected from the variation of $k_{f p l}^{\prime} / k_{f s t}^{\prime}$ with gas flow rate, as shown in Fig. 3, and agrees qualitatively with the experimental results of Schenck and Cloth ${ }^{16)}$ and Yavoǐskiī, et al. ${ }^{19)}$

\section{Influence of the Geometry of a Hematite Pellet}

The iron oxide pellet is mainly composed of hematite, and no other components contribute to the reaction. It is also assumed that during the reduction process the crack or the variations of pore structure and pore-size distribution due to the pulsation, ${ }^{16)}$ to swelling and to sintering do not occur under steady and pulsating flow, so that the unreacted-core shrinking model for one interface can be used.

\section{Pellet Diameter}

From the relations between $\Psi$ and $d_{p}$, i.e.,

$$
\begin{aligned}
& \lim _{d p \rightarrow 0}\left(\frac{\partial \Psi}{\partial d_{p}}\right)=0, \quad\left(\frac{\partial \Psi}{\partial d_{p}}\right)_{d_{p}=d_{p}^{*}}=0, \quad \lim _{d_{p} \rightarrow \infty}\left(\frac{\partial \Psi}{\partial d_{p}}\right)=0, \\
& \lim _{d p \rightarrow 0} \Psi=0 \text {, } \\
& \lim _{d_{p \rightarrow \infty}} \Psi=0
\end{aligned}
$$

the optimum pellet diameter $d_{p}^{*}$ for the reduction is expected, as shown in Fig. 7a. The variations of $\Psi$ with $d_{p}$ at given parameters are shown in Fig. 7. At $1200^{\circ} \mathrm{C}$ the optimum pellet diameter is observed as about 1 to $2 \mathrm{~cm}$. But further investigations are necessary to make more detailed discussions on the optimum pellet diameter for industrial use such as packed and fluidized beds or rotary kilns.

\section{Porosity of Unreduced Pellet}

The utility factor $\Psi$ increases as $\varepsilon_{p}$ increases irrespective of the other parameters, as shown in Fig. 8. But the degree of its increase is small, so that the porosity does not essentially contribute to the acceleration effect of pulsating flow on the reduction, according to this analysis. When the acceleration effect of pulsating flow on the reduction depends remarkably on the kinds of iron ore, or the porosity and the pore structure, it is expected that the mass-transfer rate in the particle is accelerated under pulsating flow, and therefore, further investigations are necessary.

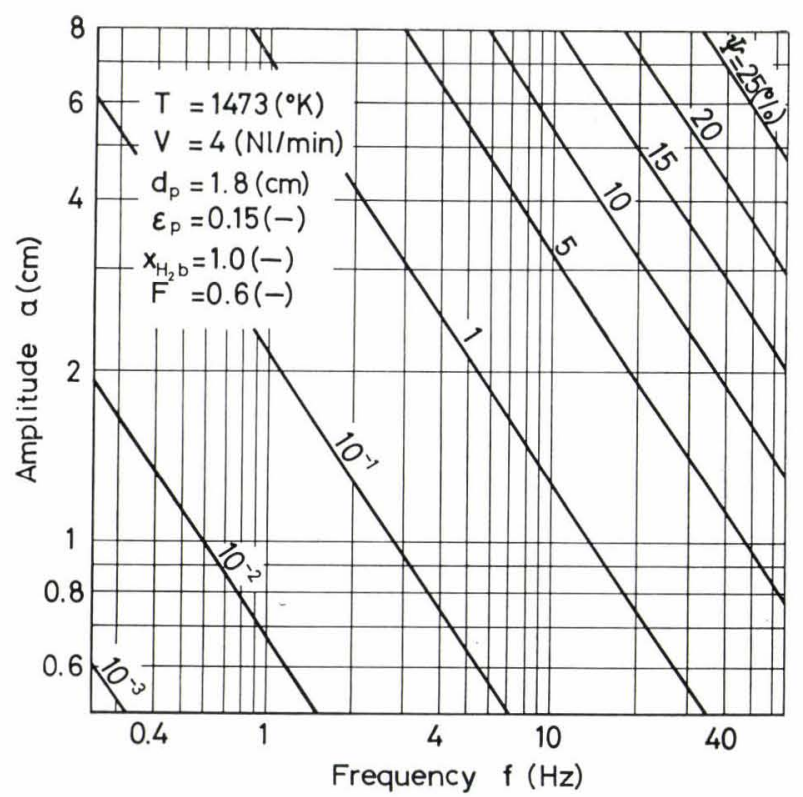

Fig. 5. Equi-utility factor lines in the $f-a$ domain

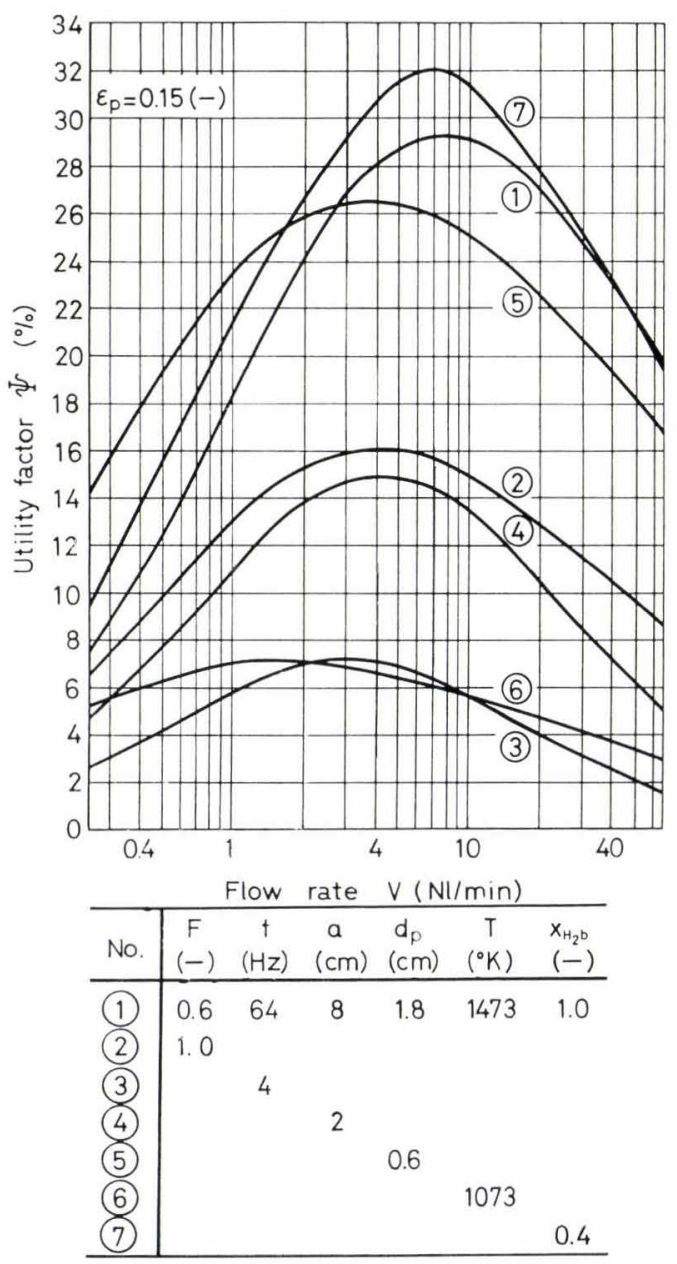

Fig. 6. Utility factor $\Psi$ as a function of time-averaged flow rate $V$

\section{Influence of Reducing Conditions}

The reaction is considered to proceed isothermally. ${ }^{33)}$ The oscillations of gas concentration and pressure under pulsating flow are assumed to be negligible small. 


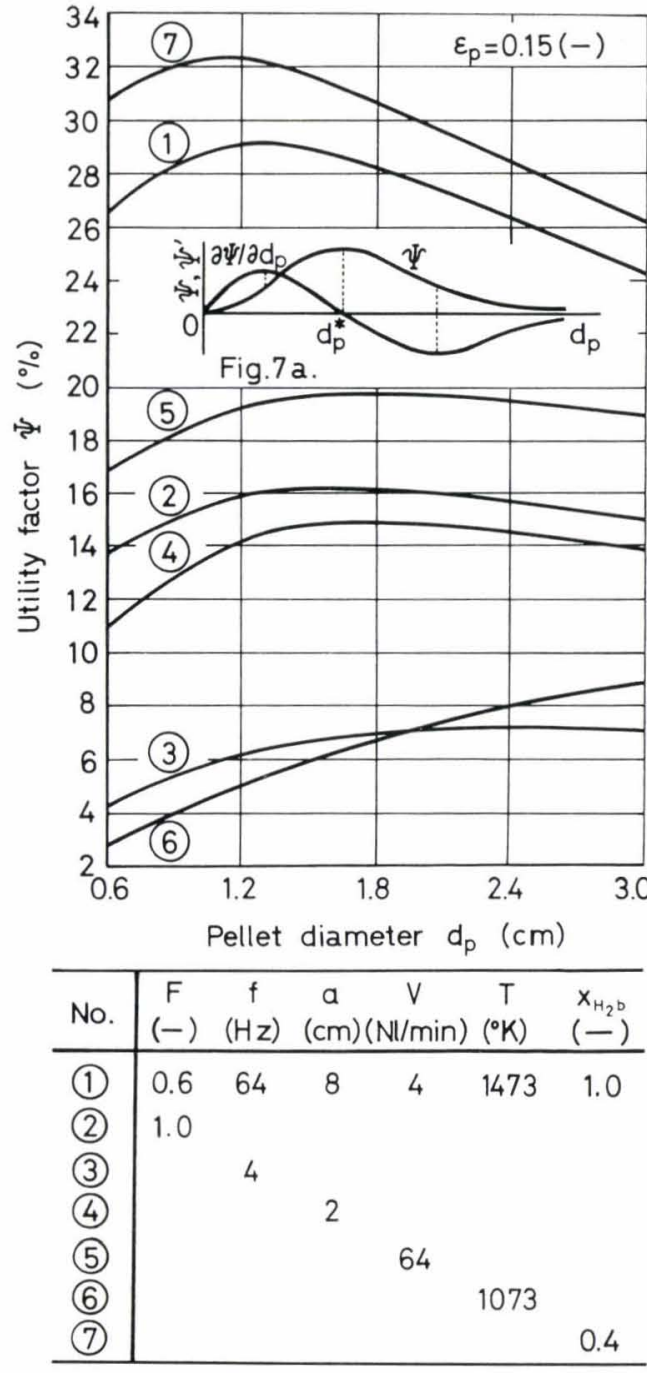

Fig. 7. Optimum pellet diameter $d_{p}^{*}$ for reduction under pulsating flow

\section{Reduction Temperature}

Figure 9 shows the variation of $\Psi$ with reduction temperature; $\Psi$ increases considerably as $\mathcal{T}$ increases irrespective of the other parameters. Without the unusual temperature dependence of reduction rate, ${ }^{34)}$ the chemical reaction rate constant increases remarkably as $T$ increases. On the other hand, the mass-transfer coefficient in the gas film, as shown in Fig. 2 (a), and the intraparticle diffusivity increase gradually as $T$ increases. Due to this reason the mass-transfer resistance in the gas film becomes large as compared with other resistances, so that the acceleration effect of pulsating flow on the reduction becomes large as $T$ increases, as mentioned by Yavoiskiī, et al. ${ }^{19)}$ on the basis of their experimental results.

\section{Mole Fraction of Hydrogen}

Figure 10 (a) shows the general variation of $\Psi$ with mole fraction of hydrogen; $\Psi$ increases gradually as $x_{\mathrm{H}_{2} b}$ increases except in the case of high flow rate (curve (5)). The investigations made at high flow rates show that $\Psi$ decreases as $x_{\mathrm{H}_{2} b}$ increases only when the pellet diameter becomes large, and that the optimum value of $x_{\mathrm{H}_{2} b}$ is observed when $d_{p}=1.8 \mathrm{~cm}$, as shown in Fig. 10 (b).

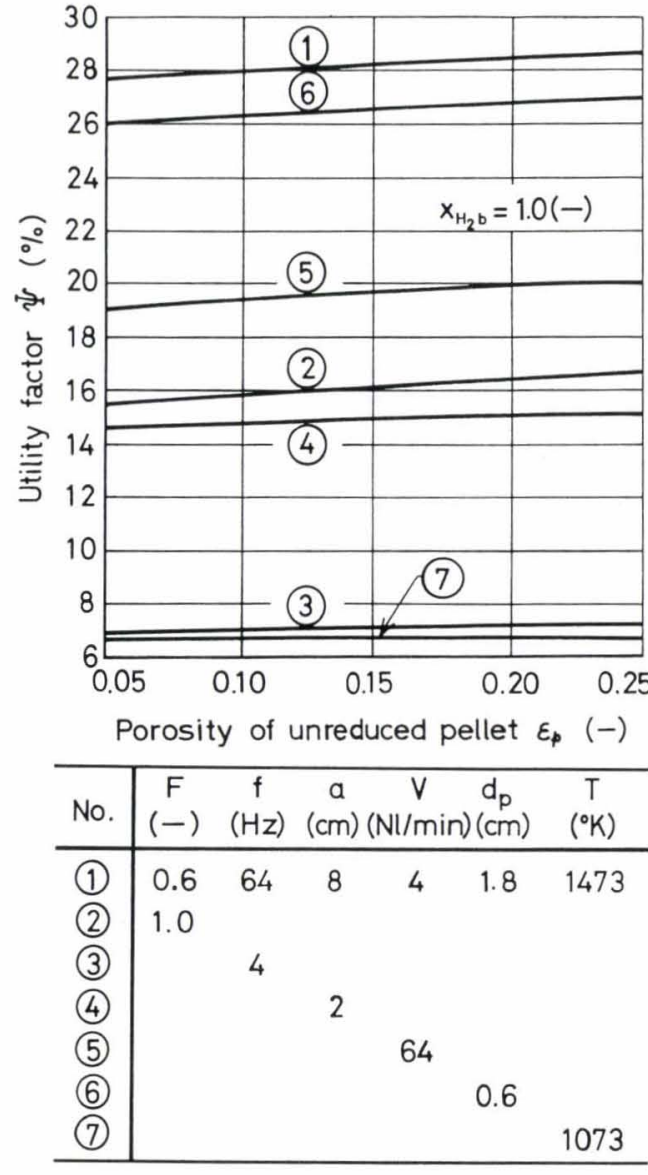

Fig. 8. Influence of porosity of unreduced pellet $\varepsilon_{p}$ on the utility factor $\Psi$

\section{Conclusions}

(1) The acceleration effect of pulsating flow on the reduction can be described clearly by the use of utility factor $\Psi$ defined as Eq. (14).

(2) The rate of reduction under pulsating flow increases as the frequency and the amplitude of pulsation increase.

(3) Pulsating flow accelerates the rate of reduction particularly in the early stage of reduction and in a high temperature range. This trend coincides qualitatively with the results previously reported. ${ }^{17-19)}$

(4) The optimum values for the pellet diameter and for the time-averaged flow rate were estimated.

(5) When the pellet diameter and the flow rate are not significantly large, the effect of pulsating flow increases gradually as the percentage of $\mathrm{N}_{2}$ gas in the binary bulk gas of $\mathrm{H}_{2}-\mathrm{N}_{2}$ increases.

(6) The utility factor $\Psi$ increases gradually as the porosity of unreduced pellet increases, according to this analysis. If the acceleration effect of pulsating flow on the mass-transfer in the particle plays an important role, further investigations will be necessary to make closer discussions.

\section{Nomenclature}

$a:$ amplitude of pulsation $(\mathrm{cm})$

$C$ : concentration of reducing gas $\left(\mathrm{mol} \mathrm{H}_{2}\right)$ 


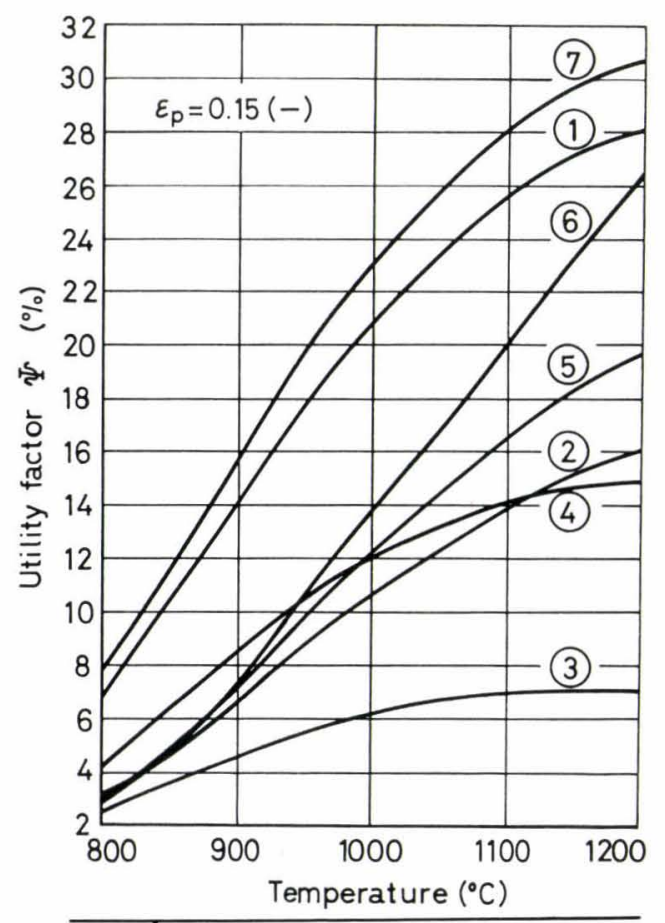

\begin{tabular}{c|cccccc}
\hline No. & $\begin{array}{c}F \\
(-)\end{array}$ & $\begin{array}{c}f \\
(\mathrm{~Hz})\end{array}$ & $\begin{array}{c}\mathrm{a} \\
(\mathrm{cm})\end{array}$ & $\begin{array}{c}\mathrm{N} / / \mathrm{min}) \\
(\mathrm{cm})\end{array}$ & $\begin{array}{c}\mathrm{x}_{\mathrm{H}_{2} \mathrm{~b}} \\
(-)\end{array}$ \\
\hline (1) & 0.6 & 64 & 8 & 4 & 1.8 & 1.0 \\
$(2)$ & 1.0 & & & & & \\
$(3)$ & & 4 & & & & \\
$(4)$ & & & 2 & & & \\
(5) & & & & 64 & & \\
(6) & & & & & 0.6 & \\
(7) & & & & & & 0.4 \\
\hline
\end{tabular}

Fig. 9. Influence of reduction temperature on the utility factor $\Psi$

$\mathrm{cm}^{3}$ )

D: inside diameter of reaction tube $(\mathrm{cm})$

$D$ : diffusion coefficient in $\mathrm{H}_{2}-\mathrm{H}_{2} \mathrm{O}$ system with $\mathrm{N}_{2}\left(\mathrm{~cm}^{2} / \mathrm{sec}\right)$

$D_{e}:$ intraparticie effective diffusivity $\left(\mathrm{cm}^{2} / \mathrm{sec}\right)$

$d_{p}:$ diameter of pellet $(\mathrm{cm})$

$F$ : fractional reduction $\equiv 1-\left(r_{i} / r_{o}\right)^{3}$

$f:$ frequency of pulsation $(\mathrm{Hz})$

$K$ : equilibrium constant for reduction reaction

$k_{c}$ : rate constant of chemical reaction $(\mathrm{cm} / \mathrm{sec})$

$k_{f j}$ : mass-transfer coefficient in gas film $(\mathrm{cm} /$ sec)

$k_{f j}^{\prime} \equiv\left(1 / k_{f j}+4 \pi r_{o}^{2} / V_{T}\right)^{-1}(\mathrm{~cm} / \mathrm{sec})$

$n$ : transfer rate of hydrogen $\left(\mathrm{molH}_{2} / \mathrm{sec}\right)$

$R e_{p}:$ Reynolds number associated with pellet diameter $\equiv u d_{p} / \nu$

$r:$ radial coordinate of pellet

Sc: Schmidt number $\equiv \nu / D$

$S h_{j}:$ Sherwood number $\equiv k_{f j} d_{p} / D$

T: absolute temperature $\left({ }^{\circ} \mathrm{K}\right)$

$t:$ reduction time $(\mathrm{sec}, \mathrm{min})$

$u$ : time-average velocity of approaching stream toward a pellet $(\mathrm{cm} / \mathrm{sec})$

$V$ : time-average gas flow rate at the normal

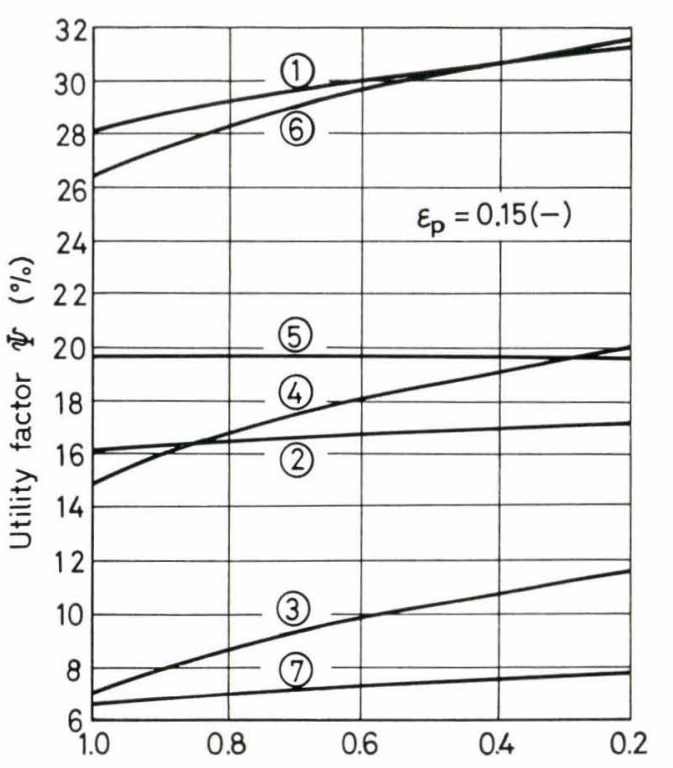

(a) Mole fraction $\mathrm{x}_{\mathrm{H}_{2} \mathrm{~b}}(-)$

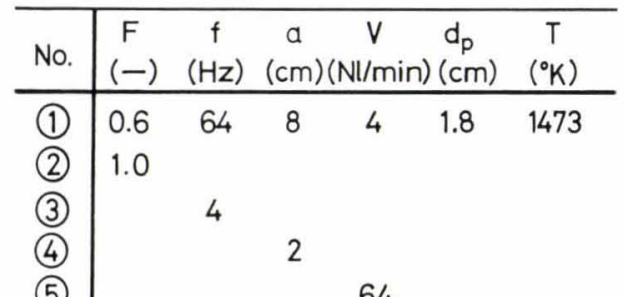

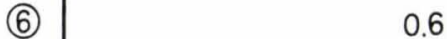

(7)

1073

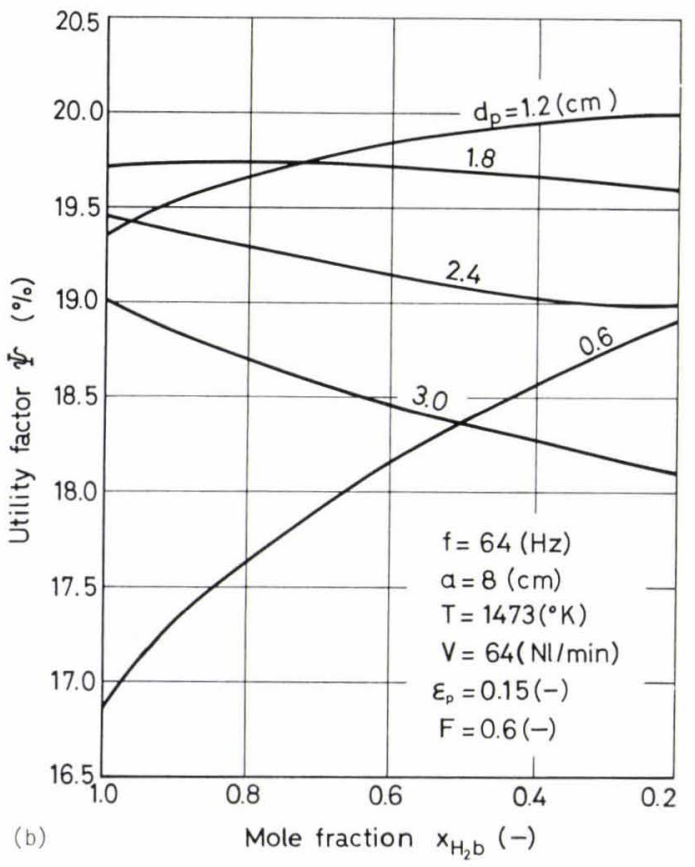

Fig. 10. Influence of mole fraction $x_{\mathrm{H}_{2} b}$ on the utility factor $\Psi$ (a) for various conditions and (b) with parameter of pellet diameter $d_{p}$

state $(\mathrm{Nl} / \mathrm{min})$

$V_{T}$ : time-average gas flow rate $\left(\mathrm{cm}^{3} / \mathrm{sec}\right)$

$x_{\mathrm{H}_{2}}$ : mole fraction of hydrogen

$\varepsilon_{p}$ : void fraction of unreacted pellet

$\nu$ : kinematic viscosity of gas $\left(\mathrm{cm}^{2} / \mathrm{sec}\right)$ 
$\rho_{p}:$ apparent molar-density of pellet (mol $\mathrm{Fe}_{2} \mathrm{O}_{3} / \mathrm{cm}^{3}$ )

$\Psi$ : utility factor of reduction under pulsating flow

\section{Subscripts}

$a, b, e, i, o:$ quantities associated with average, bulk gas, equilibrium, reaction interface and outer surface of pellet

$c, d, f:$ quantities associated with chemical reaction process, intraparticle diffusion process and diffusion process in gas film

$j=s t, p l$ : quantities for steady and pulsating flow

\section{Acknowledgements}

The authors wish to express their thanks to Mr. S. Taniguchi of Osaka University for his assistance.

\section{REFERENCES}

1) Y. Kitaura and K. Aoki: Kagaku Kōgaku, 24 (1960), 134.

2) G. J. Jameson: Chem. Eng. Sci., 19 (1964), 793.

3) P. Noordsij and J. W. Rotte: Chem. Eng. Sci., 22 (1967), 1475.

4) M. H. I. Baird: Chem. Eng. Sci., 22 (1967), 1056.

5) H. Schlichting: Boundary-Layer Theory, McGraw-Hill, New York, (1968), 411.

6) Y. Sugano and D. A. Ratkowsky: Chem. Eng. Sci., 23 (1968), 707

7) J. C. Dent: Chem. Eng. Sci., 24 (1969), 1599.

8) Y. Kitaura, H. Tanaka, G. Ueda and N. Kojima: Kagaku Kögaku, 33 (1969), 175.

9) Y. Mori, M. Imabayashi, K. Hijikata and Y. Yoshida: Int. J. Heat Mass Transfer, 12 (1969), 571.

10) H. Hara, K. Simada and K. Endoh: Kagalu Kōgaku, 35 (1971), 597.

11) M. Ohmi, T. Usui and Y. Matsumoto: Tetsu-to-Hagané, 58 (1972), S299.

12) M. Ohmi, T. Usui and Y. Kusaba: Tetsu-to-Hagané, 59 (1973), S5.

13) M. Ohmi, T. Usui and M. Sasaki: Tetsu-to-Hagané, 59
(1973), S6.

14) M. Ohmi, T. Usui and Y. Kusaba: Tetsu-to-Hagané, 61 (1975), S33.

15) M. Ohmi, T. Usui and M. Sasaki: Tetsu-to-Hagané, 61 (1975), S34.

16) H. Schenck and J. Cloth: Stahl u. Eisen, 80 (1960), 1453.

17) K. Kodama, A. Shigemi and T. Higashi: Tetsu-to-Hagané, 48 (1962), 379.

18) Y. Yamada and T. Tsuda: Tetsu-to-Hagané, 52 (1966), 1370.

19) A. B. Yavoǐskiǐ, A. A. Zhukhovǐtskiǐ and B. A. Grigoryan: Izv. VUZov., Cher. Met., (1970) No. 9, 13.

20) S. Taniguchi, M. Ohmi and M. Yamada: Tetsu-to-Hagané, 59 (1973), 1497.

21) H. W. St. Clair: Trans. Met. Soc. AIME, 233 (1965), 1145.

22) Y. Hara, M. Tsuchiya and S. Kondō: Tetsu-to-Hagané, 55 (1969), 1297.

23) M. Ohmi and T. Usui: Tetsu-to-Hagané, 59 (1973), 1888; Trans. ISIJ, 16 (1976), 77.

24) N. Frössling: Gerl. Beitr. Geophys., 52 (1938), 170.

25) M. Ohmi and T. Usui: Preprint JSME (Japan Soc. Mech. Engr.), No. 738-1, (1973), 119; Trans. JSME, 41 (1975), 546.

26) M. Ohmi and T. Usui: Preprint JSME, No. 730-6, (1973), 1; Trans. JSME, 41 (1975), 2030

27) M. Ohmi, T. Usui, M. Fukawa and S. Hirasaki: Preprint JSME, No. 735-2, (1973), 163; Trans. JSME, 41 (1975), 2043.

28) M. Ohmi, T. Usui, O. Tanaka and M. Toyama: Preprint JSME, No. 743-3, (1974), 49; Trans. JSME, 41 (1975), 2632.

29) M. Ohmi and T. Usui: Preprint JSME, No. 740-6, (1974), 1; Trans. JSME, 41 (1975), 3542.

30) M. Ohmi, T. Usui and S. Kyomen: Preprint JSME, No. 740-13, (1974), 77.

31) R. Takahashi, J. Yagi and Y. Omori: Tetsu-to-Hagané, 57 (1971), 1597.

32) Y. Hara, T. Aida and S. Kondō: J. JIM, 31 (1967), 987.

33) R. Takahashi, J. Yagi and Y. Omori: Bull. Res. Inst. Min. Dressing Met., Tohoku Univ., 27 (1971), 175.

34) M. Tokuda, H. Yoshikoshi and M. Ohtani : Tetsu-to-Haga$n e ́, 56$ (1970), 1899. 